\title{
Tendencias de investigaciones sobre prácticas de crianza en Latinoamérica
}

\author{
Trends in research on parenting practices in Latin America \\ Sandra Patricia Varela ${ }^{(0}$, Diana Castañeda ${ }^{2}$, Martha Cecilia Galindo ${ }^{3}$, \\ Ana María Moreno ${ }^{4}$, Lizeth Paola Salguero ${ }^{5}$
}

Para citar este artículo: Varela, S. P., Castañeda, D., Galindo, M. C., Moreno, A. M. Salguero, L. P. (2019). Tendencias de investigaciones sobre prácticas de crianza en Latinoamérica. Infancias Imágenes, 18(2). [247-258]

\section{Resumen}

Este artículo recoge y analiza investigaciones relacionadas con prácticas de crianza en Latinoamérica a partir de un estado del arte en el periodo 2002-2019. Se identificaron las principales tendencias actuales y las perspectivas que los estudios sugieren. El método empleado fue de revisión integrativa, cuya finalidad es obtener nuevas conclusiones a partir de resultados de investigaciones ya realizadas. La metodología fue cualitativa desde una perspectiva teórica hermenéutica a partir de estudios aplicados y teóricos que expresan modos de interrelación y tipos de prácticas parentales. Se concluye que los investigadores tienden al uso de combinaciones teóricas para comprender las prácticas de crianza, lo que da cuenta de la importancia de la educación que ofrece la familia o entorno cercano en los primeros años de vida. Se presenta un fenómeno característico: el grado de corresponsabilidad
Recibido: 12-02-2019 - Aceptado: 31-07-2019

de actores que intervienen en el desarrollo de niños y jóvenes está mediado por un factor cultural.

Palabras clave: crianza, familia, escuela, relaciones interpersonales, socialización.

\section{Abstract}

This article gathers and analyses research studies related to parenting practices in Latin America from state-of-the-art studies from 2002 to 2019. The leading current trends and perspectives that are suggested by these studies were identified. The methodology employed was one of integrative review, with the goal of arriving at new conclusions from the results of previous research studies. The methodology used was qualitative and from a hermeneutic theoretical perspective taken from academic and applied studies that advance forms of interaction and various types of parenting practices. It is concluded that researchers

\footnotetext{
Este un artículo de revisión literaria, producto de una investigación realizada en los departamentos de Casanare y Nariño de Colombia, sobre pautas y prácticas de crianza en el marco de la Maestría de Asesoría Familiar y Gestión de Programas para la Familia de la Universidad de La Sabana. Inició en febrero de 2017 y finalizó en noviembre de 2018.

1 Doctora en Educación, Universidad de Navarra, España. Profesora de la Universidad de la Sabana. Correo electrónico: sandravl@unisabana. edu.co

2 Trabajadora Social. Especialista en Gerencia Social. Especialista en Derechos Humanos y Derecho Internacional Humanitario. Magíster en Asesoría Familiar y Gestión de Programas para la Familia, Universidad de La Sabana. Correo electrónico: dianaco.82@gmail.com

3 Psicóloga. Especialista en Psicología Jurídica y Forense. Magíster en Asesoría Familiar y Gestión de Programas para la Familia, Universidad de La Sabana. Correo electrónico: martha.galindo@icbf.com

4 Psicóloga. Especialista en Gerencia Social. Magíster en Asesoría Familiar y Gestión de Programas para la Familia, Universidad de La Sabana. Docente, Secretaría de Educación de Ipiales. Correo electrónico: anamobe@unisabana.edu.co

5 Trabajadora social. Magíster en Asesoría Familiar y Gestión de Programas para la Familia, Universidad de La Sabana. Correo electrónico:
} lizethsare@unisabana.edu.co 
tend to use theoretical combinations to understand parenting practices, demonstrating the importance of what is being taught by the family or the immediate surroundings during the early years of life. A characteristic phenomenon thus emerges: the degree of co-responsibility of actors that intervene in the development of children and young people is mediated by a cultural factor.

Keywords: breeding, family, school, interpersonal relations, socialization.

\section{Introducción}

Con el paso de la historia, las formas en que padres y cuidadores ejercen prácticas con sus hijos se han ido transformando; las tendencias oscilan entre los modelos autoritarios y los permisivos. Los enfoques pedagógicos del siglo XX Ilevaron a cuestionar la coerción y el castigo físico como métodos educativos y ayudaron a desentrañar las complejidades del desarrollo infantil y sus principales vectores e hitos relacionados con la cultura, con la educación de la familia, entre otros, convirtiéndose en un campo de estudio particular e interesante.

Los pediatras, por su parte, evidenciaron la importancia de la lactancia materna por parte de madres y nodrizas; incluso, a través de la distribución masiva de cartillas de puericultura se enseñó a las madres la forma en que debían cuidar los mínimos detalles del crecimiento de sus hijos. La difusión y el control de esta enseñanza quedaron a cargo de nuevas profesionales como las visitadoras sociales y las enfermeras sanitarias.

Los fenómenos sociales también contribuyeron a cambiar las prácticas de crianza, así como también las tendencias tecnológicas, los medios masivos de comunicación, la difusión de nuevos enfoques en psicología infantil, la ampliación de la participación laboral de la mujer, la reducción en el tamaño de la familia, entre otros aspectos han variado con el paso del tiempo las prácticas de crianza en Latinoamérica.

El proceso de desarrollo holístico en las personas es determinado en gran medida por las interacciones que se dan en la familia, las instituciones educativas y la comunidad en las que se está inmerso en los primeros años de vida (Mieles y García, 2010; Silva, 2011; Meza y Páez, 2016). La combinación y unión de estas instituciones sociales implica que los niños desarrollen las habilidades, conocimientos y capacidades necesarias para su integración a un mundo social. A partir de ello, los contextos de desarrollo primarios son considerados pertinentes en la formación inicial de las personas. La labor que realizan los padres y las implicaciones que tienen son fundamentales para la integración social y el desarrollo del comportamiento de los niños (Méndez, Andrade y Peñaloza, 2013).

El estado del arte que se presenta resalta la tendencia de considerar a los padres como primeros cuidadores, lo que demuestra la importancia que tienen en el desarrollo a corto, mediano y largo plazo al ejercer una serie de actitudes, actividades y procesos de interacción guiados a promover de manera holística su desarrollo. Este tipo de actitudes se les conoce como prácticas parentales o prácticas de crianza. El estudio de estas prácticas ha implicado el interés de disciplinas como la psicología, la sociología, la neurociencia y la educación. Los resultados han demostrado que estas interacciones son vitales en el proceso de crianza y determinan de manera positiva o negativa su desarrollo en la vida adulta.

Desde investigaciones realizadas a nivel latinoamericano se evidencia que las prácticas de crianza en general son educativas y cumplen fines sociales. El objetivo de esta interrelación, de acuerdo con Ramírez es "influir, educar y orientar a los hijos para su integración social" (2005, p. 167). Es decir, en la interacción se modula la conducta de los hijos en relación con valores familiares que conducen al desarrollo de valores comunitarios.

\section{Método}

Para la revisión de las investigaciones sobre pautas y prácticas de crianza en Latinoamérica se utilizó el método de revisión integrativa (Whittemore y Knafl, 2005). Este es un método cuya finalidad es la obtención de nuevas conclusiones a partir de los resultados de otras investigaciones ya realizadas. 
Particularmente, para realizar el proceso de revisión de la literatura se siguió la metodología sugerida por Méndez et al. (2013), la cual indica que se deben seguir las siguientes etapas: delimitación del objetivo, definición de los criterios de inclusión, búsqueda de los textos en las bases de datos, análisis de los resúmenes, selección de los textos de acuerdo con los criterios de inclusión, evaluación crítica de los mismos y análisis de los datos recolectados.

El principal objetivo del estudio fue identificar las prácticas de crianza que según los estudios latinoamericanos ejercen los padres y cuidadores con los niños y niñas entre 0 y 6 años de edad. Para iniciar una revisión sistemática se partió de la pregunta generadora de conocimiento al sistematizar las investigaciones, razón por la cual se plantea: ¿qué tendencias marcan las investigaciones en cuanto a las prácticas de crianza en el periodo comprendido entre 2002 y 2019 a nivel latinoamericano?

Con el fin de limitar la revisión a los objetivos declarados y así incluir o excluir adecuadamente los textos durante el proceso, se definieron los siguientes criterios de inclusión: estudios primarios tales como artículos de revisión, artículos de investigación, artículos de prensa, estudios que reporten investigaciones relacionadas con prácticas de crianza en niños menores de 6 años, estudios de caso con prácticas parentales cuyos resultados son fiables y significativos, estudios disponibles en idiomas castellano e inglés, estudios comprendidos entre 2002 y 2019.

Con estos criterios se definió un corpus documental que incluyó artículos publicados en revistas indexadas en las siguientes bases de datos: Scopus, ProQuest y Web of Science, Redalyc, Scielo. Los descriptores de búsqueda utilizados fueron: "prácticas de crianza en... [nombre de cada uno de los países latinoamericanos]", "investigaciones sobre prácticas de crianza", "estudios de caso de prácticas de crianza en [...]", "estilos de crianza" y "pautas de crianza".

Este enfoque produjo un corpus de 350 documentos iniciales luego de revisar los títulos de los textos obtenidos con las palabras claves utilizadas en las diferentes bases de datos. Dicho corpus se redujo a 150 textos después de comparar y unificar los resultados obtenidos en las diferentes bases de datos. Para finalizar, se realizó un análisis de los resúmenes de los textos antes mencionados y se aplicaron los criterios de inclusión, lo cual limitó el corpus a los 80 textos finales incluidos en la revisión.

Para el proceso de análisis de los datos se siguió el método de análisis temático indicado por Braun y Clarke (2006), el cual consiste en los siguientes procesos: familiarización de los datos, codificación inicial, búsqueda de patrones, revisión de patrones, definición de patrones y escritura y producción del informe final.

Los 80 artículos seleccionados fueron finalmente leídos con detenimiento y se buscó la relación con la pregunta generadora que orienta la revisión, lo cual permitió la construcción de categorías de análisis emergentes.

El análisis de las categorías propició la revisión de las tendencias que existen cuando se investiga en prácticas de crianza. Se encontraron convergencias y divergencias tanto temáticas como metodológicas, lo que muestra las brechas más significativas para posibles investigaciones.

La metodología definida fue cualitativa desde una perspectiva teórica hermenéutica a partir de estudios aplicados y teóricos que expresan modos de interrelación y tipos de prácticas parentales.

\section{Resultados}

La revisión documental sistemática de las 80 investigaciones permitió la agrupación de los estudios en tres grandes categorías: principales estrategias en la crianza, los tipos de prácticas parentales y la relación entre género y crianza. A continuación, se describen estas categorías.

\section{Principales estrategias en la crianza}

Los estudios sobre prácticas parentales educativas basadas en relación con la interacción han sido desarrollados durante el último decenio dada la importancia que se ha encontrado desde el punto de vista social y psicológico (Izzedin y Pachajoa, 2009; Álvarez, 2010; Capano y Ubach, 2013; Malander, 2016; Escobar, Santamaría y Llanos, 2009; Viloria y Fernández, 2016; Villegas, Alonso, Alonso y Martínez, 2014; Varela, Chinchilla y Murad, 2015; Sanabria, 2010; Ritterbusch, 2012; 
Henao y García, 2009; Gómez y Jiménez, 2015; Dobriansyj, 2013; Cuervo, 2010; Alfaro, Chávez, Macén y Poma, 2016). Los estudios evidencian las distintas posturas conceptuales frente a las prácticas de crianza, a los contextos en los cuales se desarrolla y a la conformación -definición- de las nuevas familias, sumado a los cambios globales sobre los papeles maternos y paternos en la conformación nuclear de la familia.

En palabras de Ramírez "no se pretende decir que los padres utilicen siempre las mismas estrategias con todos sus hijos ni en todas las situaciones, sino que $[\ldots]$, dentro de un continuum más o menos amplio de tácticas, seleccionan con flexibilidad las pautas educativas" (2005, p. 168). Esto trae por lo mínimo dos supuestos: el primero, los padres utilizan una serie de estrategias, en muchas ocasiones compartidas de forma tradicional, que sirven para regular el comportamiento o la actitud de los hijos; $y$, segundo, las estrategias o prácticas utilizadas determinan elementos educativos y de interacción social, por lo que se han desarrollado como complemento en el crecimiento infantil y adolescente.

Los patrones de crianza también fueron de interés para Casais, Flores y Domínguez (2017), los cuales se enfocaron en determinar que la responsabilidad dada a los padres da cuenta de la personalidad que posee el hijo a corto, mediano y largo plazo; por otra parte, definen que las actitudes maternas responden a las creencias que se han implementado entre generaciones para orientar el comportamiento y el desarrollo de los niños. De esta manera, el desarrollo y la educación de los hijos es una prioridad para las madres en la interacción con sus hijos y con el entorno y las pautas de crianza están determinadas por las creencias intergeneracionales de los progenitores que finalmente implican unas maneras de actuar frente a sus hijos, denominadas prácticas de crianza.

Las estrategias desarrolladas por los padres, de acuerdo con Franco, Pérez y de Dios (2014), poseen un enfoque disciplinario, haciéndose más fuerte en la adolescencia, momento en el cual los padres modifican sus comportamientos y actitudes hacia los hijos de acuerdo con los cambios sociales y hormonales a los que se ve enfrentado el adolescente. De esta manera, desde edades tempranas los padres empiezan a generar comportamientos con el objetivo de establecer límites y normas. Los estudios revelan que estos últimos hacen parte del componente disciplinar, al cual se le debe sumar el afectivo, el compromiso con la crianza, la satisfacción y la percepción que tienen los padres del niño o del adolescente.

Aunque la distribución de funciones en la crianza se ha estudiado durante los últimos años, el papel de la familia como institución social encargada de los procesos de socialización, educación intencionada tiene muchos vacíos conceptuales todavía. De acuerdo con Capano, del Lujan y Massonier (2016), ese ideal de proceso depende de los estilos de la interacción que se tengan en la familia y conformación de esta; dando cuenta de la importancia de los adultos cercanos con quienes establece vínculos para formar de manera continua y compleja las manifestaciones de valores y afectos.

Para Rosabal, el nivel como se involucran los padres en la interacción puede constituir el desarrollo de una nueva atmósfera tanto social como cognitiva. En los casos en los que la intensidad de cuidadores es mayor, en el caso de las familias extensas, las estrategias de crianza permiten que "Ios niños y las niñas, desde los estadios más tempranos de su desarrollo, sean estimulados de forma simultánea" (2012, p. 84).

Finalmente, los estilos de crianza, aunque pareciesen repetirse en diferentes sociedades y contextos han sido modificados constantemente, incluso en la actualidad se establecen nuevas formas de interacción, de inculcar disciplina. Por tal motivo, y de acuerdo con los planteamientos de Franco, Pérez y de Dios (2014), no existen modelos fijos o puros de crianza, lo que implica que se presenta un dinamismo que permite que sean de carácter mixto variantes de acuerdo con variables que tienen que ver con el sexo, el género, la edad, la posición del infante/adolescente dentro del grupo de hermanos, o en el caso que sea hijo único; entre otras variables que implican la realidad familiar, social, económica y cultural.

\section{Tipos de prácticas parentales}

Las investigaciones muestran que las prácticas se dividen en dos grupos. El primer grupo tiene que ver 
con el control, la exigencia y la presión puesta por los padres; actitudes encaminadas a cumplir objetivos y metas (algunas de estas metas provienen de los ideales de los padres). El segundo grupo relacionado con las prácticas que tienen como objetivo el apoyo, la responsabilidad, la sensibilidad y la calidez, este último relacionado con aspectos sensibles de la crianza. De acuerdo con Méndez et al. (2013), la selección de estas dimensiones ha sido recogida en varios estudios, asociándolas como un apoyo parental en afectaciones a la conducta de los niños, ya que se abarca elementos tales como responsabilidad, aceptación, educación y cordialidad. Lo anterior, se sustenta en las ideas de Córdoba, quien menciona que la acción que toman los padres en la crianza "parental incide en el desarrollo cognitivo, emocional y social de niñas, niños y adolescentes dado que son las acciones y hábitos cotidianos, que padres y madres manifiestan, en respuesta a las demandas de sus hijos" (2014, p. 10).

Para Andrade, Betancourt, Vallejo, Celis y Rojas (2012, p. 2719), las prácticas parentales pueden clasificarse en tres dimensiones de medición: el afecto, el control y el apoyo. La justificación de selección se da a estudios comparativos de caso en los que a través de formulaciones y análisis cualitativos están presentes en la gran mayoría de prácticas parentales educativas.

Para los autores, el afecto es un elemento que comprende como subconjunto el apoyo, este último hace referencia al cuidado que tienen los padres frente al trato y acogida con sus hijos, para fortalecer su independencia y autonomía, las cuales se pueden identificar en actitudes hacia los hijos que estén encaminadas a niveles bajos de castigo físico, uso del pensamiento racional por padre y madre, así como el uso de una óptima comunicación. El afecto es un elemento que implica la expresión de emociones durante la interacción, lo que fomenta las relaciones entre padre-hijo calurosas, de un clima familiar agradable. Las actitudes que se relacionan con el afecto son una muy buena comunicación con los hijos de manera clara y precisa, en la que predomina el afecto, el apoyo y los vínculos emocionales.

Finalmente, el control es una exigencia de los padres hacia los hijos, cuyo fin es el de integrar a estos últimos a la familia y a la sociedad. Este tipo de crianza es frecuente en la etapa de la adolescencia, ya que a través del control se plantea modelar y mostrar una madurez, supervisar, disciplinar y fomentar la obediencia. Para Andrade, Betancourt, Vallejo, Celis y Rojas, el control puede ser de dos tipos: psicológico y conductual. El control conductual es explicado como las acciones realizadas por los padres para regular el comportamiento de los hijos. En este último elemento se rigen acciones de disciplina y supervisión. Casais, Flores y Domínguez (2017) exponen mediante su desarrollo temático y siguiendo las ideas de Baumrind (1966) y de Maccoby y Martin (1983) que hay dos dimensiones para establecer el control parental: la primera dando un cierto grado de libertad en la toma de decisiones y la segunda es la medición por parte del afecto entre padre-hijo y madre-hijo.

Por otra parte, los estudios conceptualizan las prácticas severas como las prácticas y comportamientos que son aprendidos por sus padres a raíz de la propia experiencia. A pesar de que estas prácticas son consideradas peligrosas para los niños, Berzenski y Yates (2013) afirman que la relación entre los comportamientos inadecuados de los niños y adolescentes y las prácticas de crianza son un tema de discusión de varias décadas.

Uno de los argumentos frente a este tema es que existen múltiples determinantes o variables que pueden trabajar de forma aislada (Baumrind, Larzelere y Cowan, 2002). Un ejemplo de esto es el trabajo realizado por Gershoff (2002) en el que a través de un estudio de 88 casos confirma que las prácticas severas de tipo disciplinario son un factor de riesgo para los niños y niñas. No obstante, Baumrind, Larzelere y Cowan (2002) concluyen que los estudios acerca de los mediadores potenciales que afectan las relaciones del castigo físico y las consecuencias para los niños son en su mayoría simplistas, en la medida en que es importante indagar acerca de los contextos y los métodos disciplinarios con mayor probabilidad.

Además, en un estudio realizado por Lynch et al. (2006), en el que hubo participación de cientos de padres y madres se encontró que cuando las madres o los padres usaban castigo físico severo como golpes o patadas, los problemas externalizados con 
relación a la conducta eran mayores, en comparación con las familias que no usaban este tipo de castigo. Los estudios concluyen que los problemas de conducta, los problemas emocionales, la ansiedad, la introversión o la somatización causan dificultades en la crianza de los padres, con un desapego emocional y efectuando una enseñanza en términos generales de mucha dificultad, mientras que los niños que no presentan estas dificultades y que se mantienen emocionalmente positivos generan una facilitación de la crianza y genera unos vínculos más afectivos entre los padres y los niños (Brunk y Henggeler, 1984; Goldberg, Clarke y DeIlis, 2002). En este sentido, es pertinente evaluar el comportamiento del niño, junto con todos los fenómenos que se presenten en el contexto a las variaciones en la crianza de los padres de familia (Solís, Medina y Díaz, 2013).

Otros estudios marcan tendencia en el análisis de observar a niños el comportamiento negativo desafiante y lo consideran como un patrón recurrente en las conductas de berrinche, discusión con adultos, oposición, desobediencia, irritabilidad, enojo y resentimiento hacia las figuras de autoridad, lo que causa deterioro de la actividad social, familiar o escolar (Chainé et al., 2015). Por su parte, Cornell y Frick muestran la importancia "de observar un bajo nivel de culpa por el incumplimiento de normas y un bajo de nivel de empatía con las figuras de autoridad en niños que muestran comportamientos característicos de este trastorno" (2007, p. 59). También, Chainé et al. (2015) han definido la agresión como la forma de coerción que usan los individuos para controlar las conductas de los demás (Ayala et al., 2002).

Así es como el comportamiento de orden negativo-desafiante puede reflejarse en un comportamiento antisocial que puede transgredir las normas comunitarias y familiares. En este sentido, resulta importante prevenir la progresión de las dificultades de la conducta infantil desarrollando estrategias de reducción exitosas en el comportamiento delictivo en la adolescencia. Así, la probabilidad de que un individuo se comporte de forma agresiva aumenta si se incrementa la presencia de factores de riesgo que superan a las personas que les protegen. Las investigaciones concluyen que para el caso de los niños que presentan trastornos negativistas desafiantes con bajos índices de empatía o culpa, la disciplina que basa en reglas claras "resulta ser más efectiva para que aprendan a reaccionar favorablemente a las normas establecidas por los adultos y para que su comportamiento resulte similar al de niños que reportan satisfacción por mostrar obediencia y conductas de autocontrol" (Cornell y Frick, 2007, citado por Chainé et al., 2015, p. 65)

\section{Relación entre género y crianza}

El género de los padres posee efectos en el control parental, tanto a nivel psicológico como conductual. Las investigaciones muestran que elementos como la supervivencia, el afecto y el apoyo se ven permeados de la diferencia entre géneros, ya que los padres y madres transmiten diferentes comportamientos y actitudes, en muchos casos dados por la cultura, que son útiles para el desarrollo de la identidad y de la socialización. Para García, Robles y Van Barneveld (2015) el cambio asociado al género en la crianza se manifiesta al momento en que la mujer ingresa al mundo laboral y el hombre empieza a asumir funciones domésticas, como lo son la crianza de los hijos.

Para Betancourt y Andrade (2011) la cuestión de género implica un análisis mayor, por lo que proponen determinar si realmente existe una diferencia entre prácticas parentales por parte de padres y por parte de madres, y si ha de existir si tiene relevancia en los problemas emocionales y de conducta. Los estudios documentados por Capano y Ubach (2013) han mostrado que en las familias que prevalecen pautas de crianza positivas en las cuales se rige el legado cultural de la mujer enfocado a una ética de cuidado a través de prácticas en las que prevalece la confianza, entendida como la percepción de sus hijos como personas capaces, activas y reconocidas; sin distinción entre si el hijo es masculino o femenino determinan éxito en la etapa adulta y mejores relaciones entre madre-hijo.

De acuerdo con Gallego (2012), los niños pasan por dos procesos de gestación: el primero de carácter biológico y el segundo por el contexto cultural en el cual está inmerso. "El primer proceso es trabajo exclusivo de la madre; el segundo, por otra parte, depende de la cultura en donde se desarrolla. 
Lo importante en este sentido es percibir que la madre, por ser la gestadora y cuidadora principal (esto por razones biológicas y sociales instauradas) posee una mayor responsabilidad. Para este autor, la importancia de comprender la realidad de las familias en contextos vulnerables fue fundamental, dando paso a la recolección de vivencias cotidianas para que se dé sentido a las prácticas mejoradas por las mujeres que sacan adelante a sus hijos y logran cambiar la historia y ofrecer mejor trato a sus hijos con mayores vínculos generados desde el afecto y la ternura. Como resultado de estas investigaciones, se evidencia que la interacción basada en el respeto y comprensión de las necesidades de los niños favorece el desarrollo de las capacidades sociales en la vida adulta.

Lo anterior es sustentado por los planteamientos de Méndez et al. (2013), quienes mencionan que el sexo de los padres en el ámbito familiar influye en los procesos de socialización, principalmente por el trato diferenciado con sus hijos. Las condiciones en las que viven las familias monoparentales femeninas poseen retos enormes, ya que "la crianza es un proceso que suscita limitaciones y responsabilidades, máxime cuando se habla de una mujer sin apoyo de su pareja, ama de casa, con un nivel educativo bajo, y con más de un hijo". Para Gallego, la madre es un ser fundamental encargada de la preparación de los hijos para su vida social, señala que:

Las acciones que asume la madre -como adulto responsable de la crianza- están relacionadas con el cuidado, la protección, orientación y transmisión de normas, que adicionalmente están asociadas a sentimientos que se van generando entre ella y sus hijos e hijas y que tipifican las actitudes y comportamientos que configuran el vínculo entre ambos. (2012, p. 115)

De esta manera, el vínculo expresado entre madre-hijo proviene de elementos más profundos y relacionales entre madre-mujer, generando que esto se convierta en esencial para la comprensión de las interacciones y las prácticas parentales.

Por otra parte, Méndez et al. (2013) mencionan que las madres también cumplen un papel importante, dado que los adolescentes y preadolescentes se ven afectados por la interacción que tienen con la madre, principalmente en los casos en los cuales hay devaluación y falta de comunicación por parte de esta. En estas respuestas comportamentales surge la dificultad principal, o la preocupación naciente que se tiene en relación con las prácticas parentales, ya que los padres, en este caso las madres, no saben muy bien qué estilo educativo es el adecuado para sus hijos. López, Acuña, Ponce y Tlalmis (2017) se enfocaron en estudiar las prácticas parentales de las madres con hijos con altas capacidades intelectuales que participaban en un programa de orientación. Los resultados de estas investigaciones demuestran que las relaciones afectivas, amistosas y en las que el diálogo y el razonamiento representaban un papel fundamental por parte de las madres reduce la conducta violenta de los hijos y facilita prácticas de crianza fundamentadas en el buen trato y la disciplina.

Otros estudios como los de Bolsoni (2018), plantean la interacción entre madre-hijo dando importancia a las intervenciones sociales que se desarrollan en esta y los resultados comprueban que la comunicación y el afecto por parte de las madres influyen en el comportamiento tanto positivo como negativo de los hijos e hijas. En general, las prácticas son positivas y más en aquellos casos en los que se tiene un hijo con algún tipo de enfermedad o condición especial que vulnere la realidad social.

En relación con los fenómenos histórico-sociales-humanos se encuentran investigaciones como las de Bautista (2016), en el que el papel de la mujer tras un episodio o situación de violencia permite que las prácticas sean de modo particular enfocadas a elementos de no comprensión, replicación de violencia e injusticia. La investigación tuvo lugar con mujeres víctimas de conflicto armado de Colombia; el contexto socioeducativo en el cual se desarrollan las prácticas tiene elementos particulares y variables a discutir, como las experiencias que tienen las madres-mujeres. Las mujeres que han sido expuestas a violencia desde la infancia generan comportamientos asociados con la salud mental y en el esquema cognitivo, situación que, al llegar a la adultez, propicia la replicación ese tipo de violencia en su entorno socio-familiar, siendo los más afectados sus hijos, pues sufren tratos desconsiderados, agresivos y negligentes. Según lo 
encontrado en la investigación, cuando las parejas y familiares son asesinados o desaparecidos son las mujeres quienes deben asumir la jefatura del hogar y asumen una actitud defensiva y violenta con los suyos, donde los hijos, por ser sus subordinados más cercanos, son los que sufren de modo directo la situación de indefensión.

La investigación de Escutia et al. (2014) da cuenta del papel del padre en el proceso de crianza. Esta investigación evidencia que los padres tienen algunas tareas generales que implican interacciones a partir de la cotidianidad en las que ofrecen a sus hijos reglas claras, límites, asistencia a sus necesidades básicas, acompañan su educación, además de regular las actividades cotidianas. Los resultados dan cuenta de una sociedad en la cual el hombre, como cabeza del hogar, es el encargado de marcar los límites y establecer las pautas de conducta. Este estilo de crianza está condicionado por elementos sociohistóricos en los que se ve al padre como el proveedor de protección a gran escala o de situaciones de riesgo.

Así, las investigaciones señalan que los padres también ejercen influencia sobre la forma de crianza de sus hijos; en el cual intervienes factores de personalidad; por ejemplo, si tienen carácter fuerte, son decididos ó por el contrario si son permisivos o de temperamento suave. Los estudios evidencian que los padres que son predispuestos al coraje y que muestran severidad con los niños en edad prescolar (Solís, Díaz y Rivera, 2004). Estos comportamientos o formas en la crianza tienen como resultado un incremento en la severidad de las conductas de los menores. En conclusión, los niños que presentan conducta antisocial tienden a mostrar agresión de carácter severo y crónico, así como conducta delictiva en etapas posteriores al desarrollo infantil (Cornell y Frick, 2007). Entre el 4 y el $12 \%$ de la población mundial infantil presenta problemas en términos de la conducta en algún momento de su vida. Los problemas más frecuentes se dan en niños y niñas entre los 4 y 7 años a través de la conducta negativa desafiante, la desobediencia, la conducta agresiva y la hiperactividad. Las investigaciones estiman que entre el $2 \%$ a un $16 \%$ de la población infantil del mundo ha presentado un comportamiento negativo desafiante.

Las investigaciones demuestran la importancia del papel materno en la crianza de sus hijos, pues este enfatiza de un modo u otro la conexión existente desde el proceso de gestación. Pero, dicha importancia cultural no se ha establecido hacia el padre, principalmente en aquellos contextos donde solo se considera como un elemento que provee dinero o que simplemente está ausente. Esta temática genera gran interés en investigadores y se evidencian estudios enfocados a familias monoparentales, cambiando el estado de madre protectora a madre proveedora. Es de gran utilidad establecer en futuros estudios una distinción sobre este tipo de familias en las que la madre se comporta como cabeza de hogar y en las cuales las condiciones socioeconómicas hacen que las prácticas de crianza se deleguen a otros miembros de la familia o a aquellos adultos significativos en la vida de los niños y niñas latinoamericanos.

En esta línea, las investigaciones evidencian que la comunicación entre padre-hijo y madre-hijo interfiere en el cuidado de la salud, tanto física como mental, y de otra serie de características que inciden. El papel de la madre en este sentido implica una mayor responsabilidad dados los mecanismos sociales que se establecen, ya que se ha generado como prototipo continuo de la persona encargada de las funciones esenciales de cuidado y protección, lo que implica un sentido de obligación y responsabilidad mayor que otros actores (Aguirre y Salcedo, 2016).

Otra tendencia de las investigaciones es mostrar que las acciones que ejercen los padres sobre los hijos permiten forjar conocimientos y el desarrollo de una personalidad. Es interesante evidenciar cómo las prácticas pueden variar de acuerdo con el cuidador y los cambios de comportamiento que se generan en hijos e hijas de padres divorciados y la incidencia en el desarrollo emocional y social.

\section{Conclusiones}

En la revisión documental realizada de los últimos 17 años en Latinoamérica se ha encontrado como fenómeno característico que las investigaciones 
evidencian el qué y el cómo las prácticas parentales determinan en gran medida la personalidad de un niño o adolescente, aunque siempre se hace la acotación de la importancia del factor cultural.

Las categorías emergentes de la revisión documental: estrategias de crianza, tipos de crianza y relación entre género y crianza muestran que pueden presentarse como comportamientos variados en los que se resalta la autoridad, la implementación de una norma o la formación autónoma de la personalidad en la etapa de crianza. Lo anterior implica que existe un ideal por parte de los padres enfocado al desarrollo de los jóvenes a través de sus actuaciones (la de los padres) para generar cambios que conlleven a la obediencia o restricción de elementos de la personalidad. Dichas estrategias de control se manifiestan esencialmente a través de la interacción y, por ende, la comunicación que se posee; esta es una línea de investigaciones que marcan tendencia de estudios futuros por la implicación que tienen en el proceso de crianza.

Por otra parte, las investigaciones basadas en el castigo físico coinciden en afirmar que sus prácticas de crianza son restrictivas y de mucho control y son factores de riesgo que inciden en la conducta de socialización de los niños.

Finalmente, las investigaciones evidencian un efecto de interacción entre las conductas inconscientes por parte de los cuidadores y comportamientos de baja empatía en los niños y niñas. Se encontró que el castigo físico, que se asocia a comportamientos autoritarios por parte los padres, se relaciona con la falta de empatía. Así mismo, se evidencia que los padres de los niños con alta desinhibición conductual generaron niveles altos de reactividad en sus hijos y reportes de mayores sentimientos de culpa por incumplimiento de reglas así como una mayor empatía con las figuras de autoridad.

Los principales vacíos que deben cubrir las investigaciones en el futuro se relacionan con las dinámicas y estructuras familiares, dado que estos nuevos relacionamientos generarán otras estrategias y tipos de prácticas en relación con los niños menores de 6 años.

Por otra parte, los estudios en su gran mayoría tienen métodos y enfoques predominantemente cualitativos, lo cual genera riqueza conceptual, rigor metodológico, análisis del discurso, detalles de las investigaciones y permiten mostrar la gran riqueza metodológica y diversidad cultural en las investigaciones encontradas.

Por otra parte, es interesante conocer las diferencias que surgen en dos tipos de documentos: el primero relacionado con los estudios teóricos y el segundo con los estudios empíricos. Dado que los estudios que muestran intervención generan mayores estrategias educativas para el trabajo en prácticas de crianza por parte de padres y cuidadores, lo cual permitió enriquecer la primera tendencia encontrada; mientras que los estudios teóricos dan claridad en las conceptualizaciones de los estilos, modelos y pautas en relación con la crianza.

A su vez, los documentos que se derivan de los resultados de una tesis de pregrado, de una maestría o de un doctorado evidencian estudios más contextualizados; mientras que los documentos de organismos de gobierno o instituciones como los del Instituto Colombiano de Bienestar Familiar (ICBF), Ministerio de Educación Nacional (MEN) y los del Fondo de Naciones Unidas para la Infancia (Unicef), generalmente evidencian lineamientos de las políticas enunciados desde el "deber ser" a partir de cuestiones generales sin tener en cuenta aspectos culturales y sociales, entre otros.

\section{Referencias}

Aguirre, E. y Salcedo, E. (2016). Prácticas de crianza de madres con hijos de 6 a 11 años en zona rural. En E. Aguirre, Diálogos. Discusiones en la Psicología Contemporánea (pp. 9-26). Bogotá: Universidad Nacional de Colombia. Recuperado de https://www.aacademica.org/eduardo. aguirre/10.pdf

Alfaro, S., Chávez, A., Macén, M. L. y Poma, M. A. (2016). Relación entre los estilos de crianza y la exposición a la violencia. Sawi, 3(2). Recuperado de http://revistas.unitru.edu.pe/index.php/ RSW/article/view/1448

Álvarez, M. (2010). Prácticas educativas parentales: autoridad familiar, incidencia en el comportamiento agresivo infantil. Revista Virtual Universidad Católica del Norte, 31, 253-273. Recuperado de http://revistavirtual.ucn.edu.co/ index.php/RevistaUCN/article/viewFile/44/97 
Andrade, P., Betancourt, O., Vallejo, C., Celis, O. y Rojas, R. (2012). Prácticas parentales y sintomatología depresiva en adolescentes. Salud Mental, 29-36.

Ayala, H., Pedroza, F., Morales, S., Chaparro, A. y Barragán, N. (2002). Factores de riesgo, factores protectores y generalización del comportamiento agresivo en una muestra de niños en edad escolar. Salud Mental, 25(3), 27-40.

Baumrind, D. (1966). Effects of authoritative parental control on child behavior. Child Development, 37(4), 887-907. https://doi.org/10.1111/j.1467-8624.1966.tb05416.x

Baumrind, D., Larzelere, R. E. y Cowan, P. A. (2002). Ordinary physical punishment: Is it harmful? Comment on Gershoff. Psychological Bulletin, 128(4), 580-589. https://doi. org/10.1037//0033-2909.128.4.580

Bautista, C. (2016). De víctima a victimaría: la mujer en la crianza de los hijos. Diversitas. Perspectivas en Psicología, 12, 83-96.

Berzenski, S. y Yates, R. (2013). Preschoolers' emotion knowledge and the differential effects of harsh punishment. Journal of Family Psychology, 27(3), 463-472. https://doi.org/10.1037/ a0032910

Betancourt, D. y Andrade, P. (2011). Control parental y problemas emocionales y de conducta en adolescentes. Revista Colombiana de Psicología, 20(1), 27-41. Recuperado de http://www. redalyc.org/pdf/804/80419035006.pdf

Bolsoni, A. (2018). Práticas parentais educativas na interação social mães-filhos. Revista Brasilera de Terapia Comportamental e Cognitiva, XIX(4), 25-44. Recuperado de https://www. researchgate.net/publication/323556682_Praticas_parentais_educativas_na_interacao_social_maes-filhos

Braun, V. y Clarke, V. (2006). Using thematic analysis in psychology. Qualitative Research in Psychology, 3(2), 77-101. https://doi. org/10.1191/1478088706qp063oa

Brunk, M. A. y Henggeler, S. W. (1984). Child influences on adult controls: An experimental investigation. Developmental Psychology, 20(6), 1074-1081. https://doi. org/10.1037//0012-1649.20.6.1074
Capano, Á. y Ubach, A. (2013). Estilos parentales, parentalidad positiva y formación de padres. Ciencias Psicológicas, VII(1), 8395. Recuperado de http://www.redalyc.org/ pdf/4595/459545414007.pdf

Capano, A., del Lujan, M. y Massonier, N. (2016). Estilos relacionales parentales: estudio con adolescentes y sus padres. Revista de Psicología, 34(2), 413-444. Recuperado de http://www.redalyc.org/pdf/3378/337846349009.pdf https:// doi.org/10.18800/psico.201602.008

Casais, D., Flores, M. y Domínguez, A. (2017). Percepción de prácticas de crianza: análisis confirmatorio de una escala para adolescentes. Acta de Investigación Psicológica, 2717-2726. https://doi.org/10.1016/j.aipprr.2017.06.001

Chainé, S., Romero, V., Peña, M., López, F. y Gutiérrez, J. (2015). Prácticas de crianza asociadas al comportamiento negativista desafiante y de agresión infantil. Avances en Psicología Latinoamericana. https://doi.org/10.12804/ apl33.01.2015.05

Córdoba, J. (2014). Estilos de crianza vinculados a comportamientos problemáticos de niñas, niños y adolescentes (tesis de maestría). Universidad Nacional de Córdoba, Córdoba, Argentina. Recuperado de http://lildbi.fcm.unc.edu.ar/lildbi/tesis/cordoba_julia.pdf

Cornell, A. y Frick, P. (2007). The moderating effects of parenting styles in them association between behavioral inhibition and parent-reported guilt and empathy in preschool children. Journal of Clinical Child and Adolescent Psychology, 36, 305-318. https://doi. org/10.1080/15374410701444181

Cuervo, Á. (2010). Pautas de crianza y desarroIlo socioafectivo en la infancia. Diversitas: Perspectivas en Psicología, 6(1), 111-121. Recuperado de http://www.redalyc.org/ pdf/679/67916261009.pdf

Dobriansyj, L. (2013). Estilos e prácticas parentais e sinais de depressão em adolescentes Brasileiros. International Journal of Developmental and Educational Psychology, 217225. Recuperado de http://www.redalyc.org/ pdf/3498/349852058015.pdf 
Escobar, R., Santamaría, L. y Llanos, L. (2009). Modelos mentales sobre las prácticas de crianza de algunos adultos significativos de la Escuela Normal Superior. Recuperado de http:// biblioteca.clacso.edu.ar/Colombia/alianza-cinde-umz/20130321012026/TRuthEscobarLSantamariaLdllanos.pdf

Escutia, M., Robles, E., Oudhof, H., Villafaña, L. y Garay, J. (2014). Tareas de crianza de padres mexicanos con hijos adolescentes. Ciencia Ergo Sum, 21(1), 21-26.

Franco, N., Pérez, M. y de Dios, M. (2014). Relación entre los estilos de crianza parental y el desarrollo de ansiedad y conductas disruptivas en niños de 3 a 6 años. Revista de Psicología Clínica con Niños y Adolescentes, 149-156. Recuperado de http://www.revistapcna.com/ sites/default/files/6-rpcna_vol.2.pdf https://doi. org/10.21134/rpcna.2019.06.1.5

Gallego, T. (2012). Prácticas de crianza de buen trato en familias monoparentales femeninas. Revista Virtual Universidad Católica del Norte, 37, 112-131. Recuperado de http://revistavirtual.ucn.edu.co/index.php/RevistaUCN/article/ viewFile/390/755

García, A., Robles, E. y Van Barneveld, H. (2015). Actividades de crianza y auto distribución de roles de género en una muestra de padres mexicanos. Revista Electrónica de Psicología Iztacala, 18, 495-512.

Gershoff, E. (2002). Corporal punishment by parents and associated child behaviors and experiences: A meta-analytic and theoretical review. Psychological Bulletin, 128(4), 539-579. https:// doi.org/10.1037//0033-2909.128.4.539

Goldberg, W., Clarke, K. y Dellis, E. (2002). Emotional energy as an explanatory construct for fathers' engagement with their infants. Parenting: Science and Practice, 2(4), 379-408. https://doi.org/10.1207/s15327922par0204_03

Gómez, V. y Jiménez, A. (2015). Corresponsabilidad familiar y el equilibrio trabajo-familia: medios para mejorar la equidad de género. Polis Revista Latinoamericana. Recuperado de https://journals.openedition. org/polis/10784 https://doi.org/10.4067/ s0718-65682015000100018
Henao, G. C. y García, M. C. (2009). Interacción familiar y desarrollo emocional en niños y niñas. Revista Latinoamericana de Ciencias Sociales, Niñez y Juventud, 7(2), 785802. Recuperado de http://www.redalyc.org/ pdf/773/77315614009.pdf

Izzedin, R. y Pachajoa, A. (2009). Pautas, prácticas y creencias acerca de crianza... ayer y hoy. $\mathrm{Li}$ berabit, 15(2), 109-115. Recuperado de http:// pepsic.bvsalud.org/scielo.php?script=sci_arttext\&pid=S1729-48272009000200005

López, G., Acuña, S., Ponce, D. y Tlalmis, I. (2017). Prácticas de crianza y comportamientos parentales de madres con hijos de altas capacidades intelectuales en México. En J. Núñez, M. Pérez, M. Molero, J. Gázquez, A. Barragán, M. Simón, A. Martos y C. Hernández (comps.), Perspectivas psicológica y educativa de las necesidades educativas especiales (pp. 253-259). Almería, España: Scinfoper.

Lynch, S., Turkheimer, E., Onofrio, B., Mendle, J., Emery, R. y Slutske, W. (2006). A genetically informed study of the association between harsh punishment and offspring behavioral problems. Journal of Family Psychology, 20(2), 190-198. https://doi.org/10.1037/0893-3200.20.2.190

Maccoby, E. E. y Martin, J. A. (1983). Socialization in the context of the family: Parent-child interaction. En E. M. Hetherington y P. H. Mussen (eds.), Handbook of Child Psychology: Socialization, Personality and Social Development (vol. 4, pp.1-101). Hoboken, NJ: Wiley.

Malander, N. (2016). Percepción de prácticas parentales y estrategias de aprendizaje en estudiantes secundarios. Revista de Psicología, 25(1), 1-19. Recuperado de https:// scielo.conicyt.cl/scielo.php?script=sci_arttextypid=S0719-05812016000100009

Méndez, M., Andrade, P. y Peñaloza, R. (2013). Prácticas parentales y capacidades y dificultades en preadolescentes. Revista Intercontinental de Psicología y Educación, 15(1), 99-118. Recuperado de http://www.redalyc. org/pdf/802/80225697007.pdf

Meza, J. y Páez, R. (2016). Familia, escuela y desarrollo humano: rutas de investigación educativa. Bogotá: Universidad de La Salle, Clacso. 
Mieles, M. y García, M. (2010). Apuntes sobre socialización infantil y construcción de identidad en ambientes multiculturales. Revista Latinoamericana de Ciencias Sociales, Niñez y Juventud, 8(2), 809-819. Recuperado de http:// revistaumanizales.cinde.org.co/rlcsnj/index. php/Revista-Latinoamericana/article/view/72

Ramírez, M. (2005). Padres y desarrollo de los hijos: prácticas de crianza. Estudios Pedagógicos, XXXI(2), 167-177. Recuperado de http://mingaonline.uach.cl/pdf/estped/ v31n2/art11.pdf https://doi.org/10.4067/ s0718-07052005000200011

Ritterbusch, A. (2012). Bridging guidelines and practice: Toward a grounded care ethics in youth participatory action research. The Professional Geographer, 64(1), 16-24. https://doi. org/10.1080/00330124.2011.596783

Rosabal, M. (2012). Creencias y prácticas de crianza: el estudio del parentaje en el contexto costarricense. Revista Costarricense de Psicología, 31(1-2), 65-100. Recuperado de http:// www.rcps-cr.org/openjournal/index.php/RCPs/ article/view/10

Sanabria, A. y Uribe, A. (2010). Factores psicosociales de riesgo asociados a conductas problemáticas en jóvenes infractores y no infractores. Diversidad: Perspectiva Psicológica, 6(2), 257 274. Recuperado de http://www.scielo.org.co/ pdf/dpp/v6n2/v6n2a05.pdf

Silva, I. (2011). La adolescencia y su interrelación con el entorno. Madrid: Instituto de la Juventud. Recuperado de http://www.injuve.es/sites/ defaultfiles/LA\%20ADOLESCENCIA\%20y\%20 \%20entorno_completo.pdf
Solís, P., Díaz, M. y Rivera, B. (2004). Efectos multidimensionales de un programa de crianza en la interacción recíproca de padres y sus niños con problemas de comportamiento. Psicología Conductual, 12(2), 197-2.

Solís, P., Medina, Y. y Díaz, M. (2013). Análisis comparativo de predictores potenciales de prácticas disciplinarias severas con preescolares, antes y después de un entrenamiento para padres. Acta Colombiana de Psicología, 18(2), 139-150. https://doi.org/10.14718/ acp.2015.18.2.12

Varela, S., Chinchilla, T. y Murad, V. (2015). Prácticas de crianza en niños y niñas menores de seis años en Colombia. Zona Próxima, 22, 195-215. Recuperado de http://rcientificas.uninorte.edu. co/index.php/zona/article/viewArticle/6129 https://doi.org/10.14482/zp.22.6129

Villegas, M. Á., Alonso, M. M., Alonso, B. A. y Martínez, R. (2014). Percepción de crianza parental y su relación con el inicio del consumo de drogas en adolescentes mexicanos. Aquichan, 14(1), 41-52. Recuperado de http://www.redalyc.org/pdf/741/74130041005.pdf https:// doi.org/10.5294/aqui.2014.14.1.4

Viloria, C. y Fernández, A. (2016). Las prácticas de crianza de los padres: su influencia en las nuevas problemáticas en la primera infancia. Revista Nacional e International de Educación Inclusiva, 9(1), 30-42.

Whittemore, R. y Knafl, K. (2005). The integrative review: Updated methodology. Journal of $\mathrm{Ad}$ vanced Nursing, 52(5), 546-53. https://doi. org/10.1111/j.1365-2648.2005.03621.x

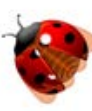

\section{(C) $(\otimes \odot \odot$}

Esta obra está bajo licencia Creative Commons Atribución-NoComercial-Compartirlgual 4.0 Internacional https://creativecommons.org/licenses/by-nc-sa/4.0/deed.es

ISSN 1657-9089 • Vol 18, No 2 (julio-diciembre 2019). pp. 247-258 